disease activity and active disease, there was no difference in BAFF/APRIL levels between groups. Serum BAFF levels were higher in patients with renal disease activity (median 0.94 $\mathrm{ng} / \mathrm{ml}$ vs $0.61 \mathrm{ng} / \mathrm{ml}, \mathrm{p}=0,01$ ), and there was a positive correlation between APRIL levels and proteinuria $(r=0.42$, $p=0,02$ ). There was no association between BAFF/APRIL levels and anti-dsDNA positivity but a weak inverse correlation was observed between BAFF and C3 levels $(r=0.25$, $\mathrm{P}=0.02)$. No correlation was found between BAFF/APRIL levels and renal SLEDAI scores, histopathologic activity and chronicity index scores. In the active disease group after follow-up, there was no significant changes in BAFF (from 1,63 $\mathrm{ng} / \mathrm{ml}$ to $1,2 \mathrm{ng} / \mathrm{ml}$ ) and APRIL levels (from 2,11 $\mathrm{ng} / \mathrm{ml}$ to $2,31 \mathrm{ng} / \mathrm{ml})$.

Conclusions BAFF/APRIL levels were found to be significantly higher in patients with SLE compared to controls, but no association with disease activity was found. BAFF levels are correlated with decreased C3 levels. These results suggest that both cytokines are involved in the pathogenesis of SLE, and that serum BAFF and APRIL levels can be valuable biomarkers in SLE especially in patients with renal activity. Long-term studies on the effect of treatment are needed.

Acknowledgements This work was supported by Scientific Research Project Coordination Unit of İstanbul University (Project number: TTU-2016-22314).

\section{P27 MYXOVIRUS RESISTANCE PROTEIN A IS A USEFUL ADDITIONAL HISTOLOGICAL MARKER FOR CUTANEOUS LUPUS ERYTHEMATOSUS}

${ }^{1}$ Wietske M Lambers, ${ }^{2}$ Gilles FH Diercks, ${ }^{3}$ Femke M Homan, ${ }^{1}$ Berber Doornbos-van der Meer, 'Hendrika Bootsma, 'Johanna Westra, ${ }^{1}$ Karina de Leeuw. ${ }^{1}$ Dept. of Rheumatology and Clinical Immunology, University Medical Centre Groningen, University of Groningen; ${ }^{2}$ Dept. of Pathology, University Medical Centre Groningen, University of Groningen; ${ }^{3}$ Dept. of Dermatology, University Medical Centre Groningen, University of Groningen, Groningen, The Netherlands

10.1136/lupus-2020-eurolupus.75

Background Cutaneous Lupus Erythematosus (CLE) is a heterogeneous auto-inflammatory skin disease, that is to a great extent driven by type I and III interferon (IFN). Histology of skin biopsies plays an important role in the diagnostic confirmation of CLE. Unfortunately, no specific histological marker for CLE is available. In this study, we tested the diagnostic potential of immunostaining with Myxovirus resistance protein A (MxA), which is tightly induced by type I and type III IFN, in CLE skin biopsies.

Methods 178 skin biopsy specimens were collected from the local pathology database. Various skin conditions were selected, provided that clinical diagnosis matched with histological diagnosis. Skin sections were incubated with anti-MxA (R\&D systems, AF7946). Consecutively, rabbit anti goat-HRP conjugate (Dako, 0449) was added and sections were stained with diaminobenzidine. The expression of MxA was scored semi-quantitatively.

Results MxA staining was strongly positive in $90.3 \%$ of lesional CLE skin sections (except lupus tumidus) and had a negative predictive value of $94 \%$. The same MxA expression pattern was found in dermatomyositis, which is also an IFNdriven autoimmune disease. In some conditions, like perniosis and graft versus host disease, high expression could be found,

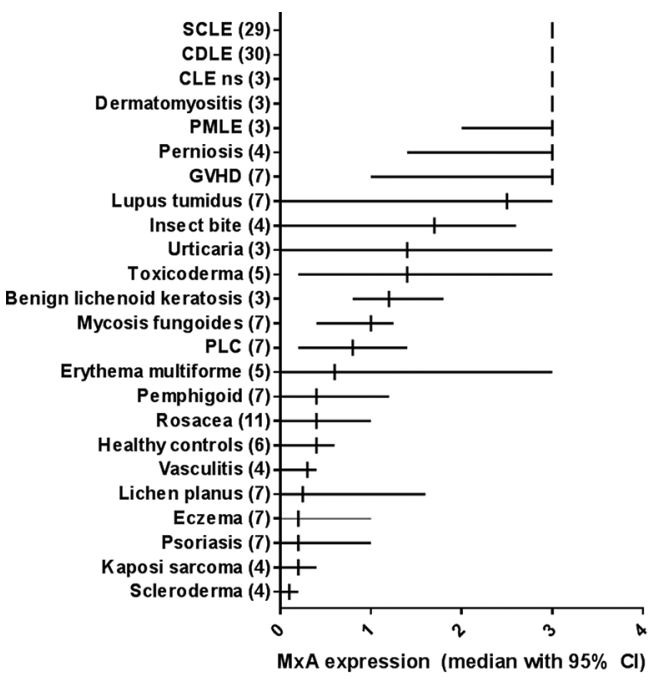

Abbreviations: $n s=$ not specified, $S C L E=$ subacute cutaneous lupus erythematosus, CDLE = chronic discoid lupus enythematosus, PMLE = polymorphic light eruption, GVHD $=$ graft versus host disease, PLC $=$ pityriasis lichenoides chronica

Abstract P27 Figure 1 MXA expression in all analyzed skin diseases (number of biospsies)

but this was less consistent compared to CLE. Most other inflammatory skin diseases did show no or a low expression of MxA. (See figure 1).

Conclusion MxA is strongly expressed in CLE skin with a high negative predictive value and is thus useful as additional diagnostic histological marker, expectedly resulting in restriction of misdiagnosis and treatment delay.

\section{P28 DECREASED PLATELET SIZE IN SYSTEMIC LUPUS ERYTHEMATOSUS IS ASSOCIATED WITH UP- REGULATION OF TYPE I INTERFERON PROTEINS}

${ }^{1}$ Petrus Linge, 'Lina Wirestam, 'Sabine Arve, ${ }^{2}$ Robin Kahn, ${ }^{1}$ Andreas Jönsen, ${ }^{1}$ Anders Bengtsson. 'Dept. of Clinical Sciences Lund, Section of Rheumatology, Lund University, Lund; ${ }^{2}$ Dept. of Clinical Sciences Lund, Section of Paediatrics, Lund University, Lund, Sweden

\subsection{6/lupus-2020-eurolupus.76}

Background Dysregulated apoptosis is of major importance in Systemic Lupus Erythematosus (SLE) pathogenesis, linked to the development of autoantibodies, immune complex formation and type I interferon signaling. Platelets from SLE patients are smaller in size, compared to platelets from healthy individuals, which may suggest an increased rate of apoptosis, a known cause of platelet shrinkage. Our aim with this project was to investigate if decreased platelet size could be explained by increased apoptosis rates.

Methods Platelet activation markers; CD62P, CD41, CD154, CD32, PAC-1 and PAR1 and apoptosis; Annexin V, Caspase 3 activation, mitochondrial content (MitoTracker) and mitochondrial depolarization (JC-1) where analyzed in 23 SLE patients and 10 healthy controls (HC) by flow cytometry. Analysis of the total protein content in platelets from SLE patients of normal $(n=5)$ and decreased $(n=5)$ size were made using mass spectrometry (MS).

Results The level of CD41 $(\mathrm{p}=0.001)$ positive platelets and mean expression of CD154 $(\mathrm{p}=0.004)$ were higher in SLE patients. A JC-1 ratio $(\mathrm{p}=0.0001)$ indicting increased mitochondrial depolarization was significantly associated with 